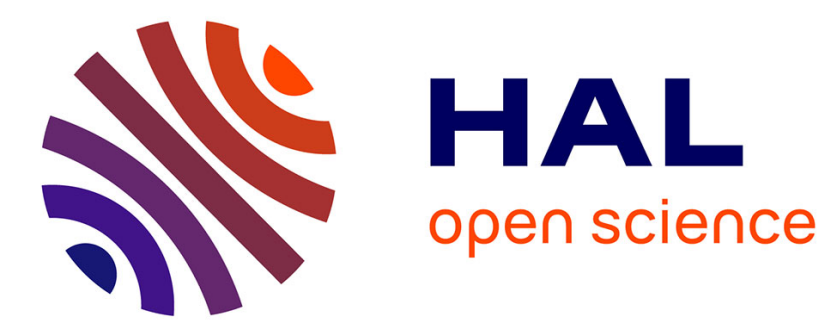

\title{
RA2DL: New Flexible Solution for Adaptive AADL-based Control Components
}

Farid Adaili, Olfa Mosbahi, Mohamed Khalgui, Samia Bouzefrane

\section{To cite this version:}

Farid Adaili, Olfa Mosbahi, Mohamed Khalgui, Samia Bouzefrane. RA2DL: New Flexible Solution for Adaptive AADL-based Control Components. 5th international conference on Pervasive and embedded computing and communication systems, Feb 2015, Angers, France. pp.63-77. hal-02425179

\section{HAL Id: hal-02425179 \\ https://hal.science/hal-02425179}

Submitted on 29 Dec 2019

HAL is a multi-disciplinary open access archive for the deposit and dissemination of scientific research documents, whether they are published or not. The documents may come from teaching and research institutions in France or abroad, or from public or private research centers.
L'archive ouverte pluridisciplinaire HAL, est destinée au dépôt et à la diffusion de documents scientifiques de niveau recherche, publiés ou non, émanant des établissements d'enseignement et de recherche français ou étrangers, des laboratoires publics ou privés. 


\title{
RA2DL: New Flexible Solution for Adaptive AADL-based Control Components
}

\author{
Farid ADAILI ${ }^{1,2}$, Olfa MOSBAHI ${ }^{1}$, Mohamed KHALGUI ${ }^{1}$ and Samia BOUZEFRANE ${ }^{3}$ \\ ${ }^{1}$ LISI Laboratory, INSAT, University of Carthage, Tunisia \\ ${ }^{2}$ Tunisia Polytechnic School, University of Carthage, Tunisia \\ ${ }^{3}$ Conservatoire National des Arts et Métiers, France \\ Email: \{adaili.farid, olfamosbahi, khalgui.mohamed\}@gmail.com and samia.bouzefrane@cnam.fr
}

\begin{abstract}
The paper deals with adaptive component-based control systems following the Architecture Analysis and Design Language (denoted by AADL). A system is assumed to be a network of software and hardware AADL components that share the control of corresponding physical processes. A component is composed of a set of algorithms encoding the control after any reception of external events and data signals. The termination of execution is generally done with the emission of data and event signals to remote components. According to various evolutions in environment, the system is required to be dynamically reconfigured at run-time to adapt its control functions. We are interested in local reconfigurations of components dealing with the activation-deactivation-update of algorithms and/or data-event inputs and outputs. We propose RA2DL as a solution for reconfigurable AADL components, and define a hierarchical-based architecture to dynamically handle all possible reconfiguration scenarios at run-time. We model and verify this solution and develop a tool for its simulation by taking a real-case study as a running example.

Index Terms-Control System, Component, AADL, Reconfiguration, Modelling, Verification, Simulation, Radar.
\end{abstract}

\section{INTRODUCTION}

Embedded control systems [1] continue to grow exponentially and has become critical and complex under usually functional and temporal constraints to be described in user requirements [2]. According to various evolutions of the environment due to incidents or also optimization of performance, the system is required to be flexible by adapting its behavior at run-time. Nevertheless, this adaptation is not easy to be done since it should generally preserve the system safety while meeting its constraints. Nowadays, two reconfiguration policies exist, (i) static reconfiguration [3] to be generally applied offline: (ii) and dynamic reconfiguration that can be applied at run-time. We generally define two solutions for the second case: manual reconfigurations to be applied by users at run-time [4], and automatic reconfigurations which are generally handled by software autonomous agents [5]. We are interested in this paper in automatic reconfigurations of embedded control systems. In order to reduce their development and consequently their time to market, these systems are based on the componentbased approach [6]. A component is classically defined as a software unit to be composed with others in order to form the general control functions of the whole system [7]. Two families of components are proposed: the components to be composed at run-time such as .Net [8], COM-DCOM [9],
Enterprise JavaBeans [10], and the components that should be composed off-line to check their respect of functional and temporal constraints such as IEC61131 [11], IEC61499 [12], Metah [13], ACME [14], Rapide [15], Wright [16], Aesop [17] and AADL [18]. We are interested in this paper in the AADL technology. AADL component is a software unit to be encoded with a set of algorithms that implement its control functions. Each algorithm is activated by corresponding external event-data inputs, and generally produces the results of its execution on corresponding data-event outputs. It is well-used in many industrial applications such as Avionics Software [19], Harmony System Engineering (Harmony-SE) [20] and M2M [21]. We note that a rich library is available today to develop applications in this technology. Nevertheless, these applications are not flexible and cannot be adapted to their environment since SAE (Society of Automotive Engineers) [22] does not provide technical solutions for the possible adaptation of the system based on AADL components at run-time. Moreover, no one in all related works deal with the flexibility of AADL components. We propose in this paper a new concept of reconfigurable AADL components to be named RA2DL that allows (1) the activation-deactivation of algorithms at run-time in order to adapt the control functions, (2) the activation-deactivation of the corresponding data-event inputs-outputs, (3) the adaptation of the execution traces (scheduling) of algorithms in the component, (4) the light reconfiguration of data according to user requirements. In order to control the complexity of the problem, we propose a control unit-based architecture to apply local reconfiguration scenarios in a RA2DL component.

We are interested in this research work in automatic reconfigurations of RA2DL components which are composed of two Modules: Controller Module that handles these reconfigurations according to user requirements and also the run-time evolution of the environment; and the Controlled Module that represents all the different services offered by the component. These services are reconfigurable and implemented by different algorithms to be activated by external event-data inputs before providing results on corresponding event-data outputs. To cover all possible reconfiguration forms while controlling their complexity, we specify the Controller Unit in three levels (i) Architecture level that creates/removes or updates algorithms or 
input/output data/event, (ii) Composition level that updates compositions of their internal behaviors and (iii) Data level that applies light reconfigurations by data. The Controller Module is modelled by Nested State Machines where states of a machine correspond to other state machines. We use the well-known environment UPPAAL [23] to model and verify the correctness of RA2DL components. The paper's contribution is applied to a case study of a radar system that will be followed as a running example. This system is deployed on an Arduino microcontroller, and a tool named RA2DL tool is developed in our LISI Lab at University of Carthage in Tunisia to implement and simulate this case study.

We present in the next section the Architecture Analysis and Design Language (AADL), and define in Section 3 the case study of the radar system. Section 4 proposes the concept of RA2DL, and Section 5 defines the modelling and verification where an UPPAAL-based model checking is applied. We propose in Section 6 an implementation and simulation of RA2DL tool and conclude the paper in section 7.

\section{AADL}

The Architecture Analysis and Design Language (AADL) [24] is an architecture description language used to model the software and hardware architecture of an embedded, real-time system. Due to its emphasis on the embedded domain, AADL contains constructs for modeling both software and hardware components (with the hardware components named "execution platform" components within the standard). This architecture model can then be used either as a design documentation, for analyses (such as schedulability and flow control) or for code generation (of the software portion) ( version 1.0 released in 2004 [25] and version 2.0 released at the end of 2009. [26]). Within the AADL, a component is characterized by its identity (a unique name and runtime essence), possible interfaces with other components, distinguishing properties (critical characteristics of a component within its architectural context), and subcomponents and their interactions.

AADL defines several categories of components, divided into three categories:

1) Software Components: (i) Data: represent data structures which can be stored or exchanged between components, (ii) Sub-programs: represent fragments of executable sequence codes, such as call-return and calls-on methods, (iii) Process: defines memory spaces in which threads are running, (iv) Threads: active components that can execute concurrently and be organized into thread groups. They can be compared with light processes as defined in the operating systems, (v) thread group: component abstractions for logically organizing threads, data, and groups of thread components within a process,

2) Hardware Components: (i)Processor:schedules and executes threads, (ii) Memory:stores code and data, (iii) Bus: interconnects processors, memory, and devices, (iv) Device: represents sensors, actuators, or other components that interface with the external environment,

3) System:design elements that enable the integration of other components into distinct units within the architecture

The AADL can be used to model and analyze systems already in use and design and integrate new systems. The AADL can be used in the analysis of partially defined architectural patterns (with limited architectural detail) as well as in full-scale analysis of a complete system model extracted from the source code (with completely quantified system property values). AADL supports the early prediction and analysis of critical system qualities, such as performance, schedulability, and reliability. For example, in specifying and analyzing schedulability, AADL-supported thread components include the predeclared execution property options of periodic, aperiodic (event-driven), background (dispatched once and executed to completion), and sporadic (paced by an upper rate bound) events. These thread characteristics are defined as parts of the thread declaration and can be readily analyzed.

An AADL model specifies how the different components interact and are integrated to form a complete system. The AADL standard also describes the run-time mechanisms for handling messages and events, synchronized accesses to shared resources, and thread scheduling when several threads run on the same processor. AADL participates in several industrial applications such us avionics industry, [19], transport system [27], Harmony System Engineering (Harmony-SE) [20], M2M (Machine-to-Machine) platform [21], ASSERT project ${ }^{3}$ [28]. We are interested in this technology because it has useful advantages: AADL offers the possibility to describe the complete hardware/software architecture of embedded control systems, it responds to architectural constraints and can represent multi-modal systems. AADL Standard prescribes the rules for activation and deactivation of components during a mode switch, and a rich library is available today pushing to reuse applications based on AADL. Nowadays, various books deal with this language. Various sophisticated tools are completely deployed according to this technology: Stood [29] also introduces some methodological features to facilitate the operational use of the AADL within industrial projects, OSATE [30] targets both end users and tool developers. The former provides a complete textual editor for AADL and a set of simple analysis tools while the latter provides a full support for the AADL meta-model on an Eclipse platform. TOPCASED [31] is a software environment primarily dedicated to the realization of critical embedded systems including hardware and/or software. ADes [32] makes possible the evaluation and analysis of the behavior of a system during its specification with AADL, for instance by helping in the choice of dimensioning parameters: what will happen if we enlarge an execution time? if we change a deadline? if we bind a task on another processor?, Ocarina [33] is an AADL 
tool that generates codes from AADL models. It runs on Linux, Mac OS X, Windows and Solaris. ADELE [34] has been created to provide new versions of ADELE editor and also Osate2 feature. Cheddar [35] is a free real-time scheduling tool. Although these tools are useful, they do not provide solutions to develop flexible AADL components for adaptive embedded systems. We mean by flexibility the facility to change the behavior of a component according to user requirements and evolution of the environment. The current paper proposes new solutions to allow reconfigurable AADL components called RA2DL which are assumed to be adaptive at run-time according to user requirements.

\section{CASE STUDY: AADL-BASED COMPONENTS FOR A RADAR SYSTEM}

We use as a running example in the current paper an AADL-based radar represented by the STOOD tool [36] as shown in Fig.1. As described in [37] and detailed as an archive of Ocarina ${ }^{1}$, the radar is composed of the following AADL components: (A) Hardware components represented by (i) an Antenna component which is a device that simulates the radar environment, (ii) Processor component which is a part of the execution platform, (iii) Memory component which hosts the address spaces, (iv) Bus component that ensures the communication between the antenna and the main process stored in memory, (v) Motor component which is a device to rotate constantly the antenna and returns the angle. (B) Software components assigned to the processing component which is composed of the following threads:

$$
\begin{gathered}
\text { transmitter } \rightarrow \text { angle_controller } \rightarrow \text { receiver } \rightarrow \\
\text { analyser } \rightarrow \text { display. }
\end{gathered}
$$

Where: (i) transmitter: a thread that sends the radar signals to the antenna, (ii) angle_controller: a thread that computes the angle of the radar, (iii) receiver: a thread that receives any information from the antenna, (iv) analyser: a thread that compares the transmitted and received signals to perform the detection, localization and identification of objects, finally (v) display: a thread that displays the objects on the radar screen. The processing component has two data inputs: (i)get_angle: from the motor position, and (ii) receive_pulse: from the target detected object. It has also two event outputs: (i) to_screen, and (ii) send_pulse. Each internal thread has also data/event inputs and outputs to support its interaction with remote threads. The reader can find more details on this radar in [37]. Although this system is well-tested, it lacks any possible flexibility that can adapt its behavior at run-time when faults occur, or when the radar environment evolutes and requires useful changes in the system's behavior. This flexibility is well-required for modern systems and represents a new challenge for the radar case study. Let us expose some reconfiguration scenarios that can adapt the radar to its environment at run-time. Let us suppose that the radar sends $M$ pulses and detects $N$ objects at a particular time. Let us denote also by (i) $p_{i}$

\footnotetext{
${ }^{1}$ http://aadl.telecom-paristech.fr
}

the $i-t h$ pulse $(i \in[1, M])$ to be sent from the antenna with a frequency $f_{i}$, (ii) $O_{j}$ the $j-t h(j \in[1, N])$ detected object from the radar. It is characterized by a direction $r_{i}$, a distance $d_{i}$ from the radar, and a surface $s_{i}$, (iii) $C$ is a radar static parameter to be used for the processing of areas in $m^{2}$. It is equal to $H_{-} R e s$ if the radar runs with a high resolution, otherwise $L_{-} R e s$ if with low resolution, (iv) condition_weather a boolean parameter which is equal to 0 when the weather is bad (snowing or running), and (v) wind_speed which represents the wind speed. We assume that the radar has two motors $M 1$ and $M 2$ to rotate the antenna with two speeds according to the wind speed. Each motor is controlled by a corresponding software AADL component. We assume in the current paper that we have two threads allowing the emission of pulses with two periods according to the weather conditions: the first sends the pulses each $6 \mathrm{~ms}$ whereas the second each $2 \mathrm{~ms}$. We note that the calculation of the angle can be done before the reception of signals or also after that if we want to optimize the performance of the radar. The calculation of the angle before the reception of signals is done when the traffic is low, otherwise it should be done each time a pulse is sent from the antenna.

1) Reconfiguration 1: If there exists an object $O_{j}(j$ $\in[1, N])$ such that $s_{j}<C$, Then the processing component reconfigures the parameter $C$ from $L_{-}$Res to $H_{-}$Res to allow a possible detection of the object,

2) Reconfiguration 2: If condition_weather $==1$, Then the pulses are periodically sent from the antenna by a thread $E V \_T 1$ each $6 \mathrm{~ms}$,

3) Reconfiguration 3: If condition_weather $==0$, Then the pulses are periodically sent from the antenna by a thread $E V \_T 2$ each $2 \mathrm{~ms}$,

4) Reconfiguration 4: If wind_speed $>100 \mathrm{~km} / \mathrm{h}$, Then the first radar motor $M 1$ rotates $45 \mathrm{tr} / \mathrm{mn}$. We assume in this case that a particular software AADL component Rotat1 is executed to control the first motor,

5) Reconfiguration 5: If wind_speed $<100 \mathrm{~km} / \mathrm{h}$, Then the second radar motor $M 2$ rotates $30 \mathrm{tr} / \mathrm{mn}$. We assume in this case that a second software AADL component Rotat 2 is executed to control the second motor.

Although AADL is a well-expressive language, it lacks useful technical solutions for the reconfiguration of hardware and software components at run-time. We propose in this paper to enrich this important language with new solutions in order to allow more flexible components that can be reconfigured at run-time. We focus in this paper on the reconfiguration of the AADL software Pocessing Component which includes a set of sub-components (algorithms).

\section{RA2DL: RECONFIGURATION OF AADL}

\section{A. Motivation: Reconfiguration Forms}

We define in this section a new concept named $R A 2 D L$ as a solution for reconfigurable AADL components where the 


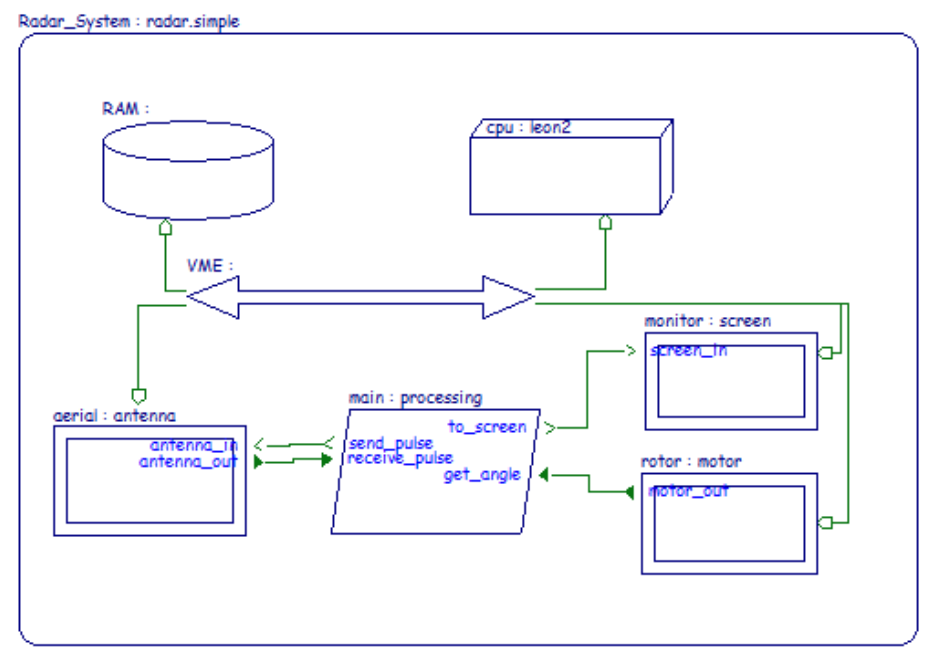

Fig. 1. Graphical AADL representation of a radar components [37]

interface of the AADL component contains data/event inputs and outputs supporting interactions with the environment. Events are responsible for the activation of the algorithms while data contain valued information of the AADL component. RA2DL is proposed in the current paper to adapt the AADL to its environment at run-time.

Throughout our study, we concentrate on three hierarchical reconfiguration levels that we present in the following:

(i) Form 1: Architectural Reconfiguration: modifies the component architecture when particular conditions are met. This is done by adding new algorithms, events and data or removing existing operations in the internal behaviors of the component. (ii) Form 2: Compositional Reconfiguration: modifies the composition of the internal components (algorithms) for a given architecture. (iii) Form 3: Data Reconfiguration: changes the values of variables without changing the component algorithms.

\section{B. RA2DL Architecture}

We define a new architecture for a $R A 2 D L$ component (to be denoted by $\mathrm{Cmp}$ ). This architecture is composed of a Controller module and a Controlled module, where the first one is a set of reconfiguration functions applied in RA2DL, and the second one is a set of input/output events, algorithms, and data as represented in the four reconfiguration modules $R M$ in Fig.2:

- $\quad$ IEM (Input Events Module): This module processes the reconfiguration of input events $(I E)$ stored in the $I E D B$ database of input events. It defines and activates at a particular time a subset of events to execute the corresponding algorithms in RA2DL.

- OEM (Output Events Module): This module processes the reconfiguration of output events $(O E)$ stored in the $O E D B$ database of output events. It defines and activates at a particular time a subset of events to be sent once the corresponding algorithms finish their execution in RA2DL.
- $\quad A L M$ (Algorithms module): This module processes the reconfiguration of the active algorithms (addition or removal) at a particular time in order to be coherent with active input and output events of $I E M$ and $O E M$. These algorithms are stored in the $A L D B$ database of algorithms.

- $\quad D M$ (Data Module): This module processes the reconfigurations of data in RA2DL in coherence with the rest of modules. It is stored the $D D B$ database of data values.

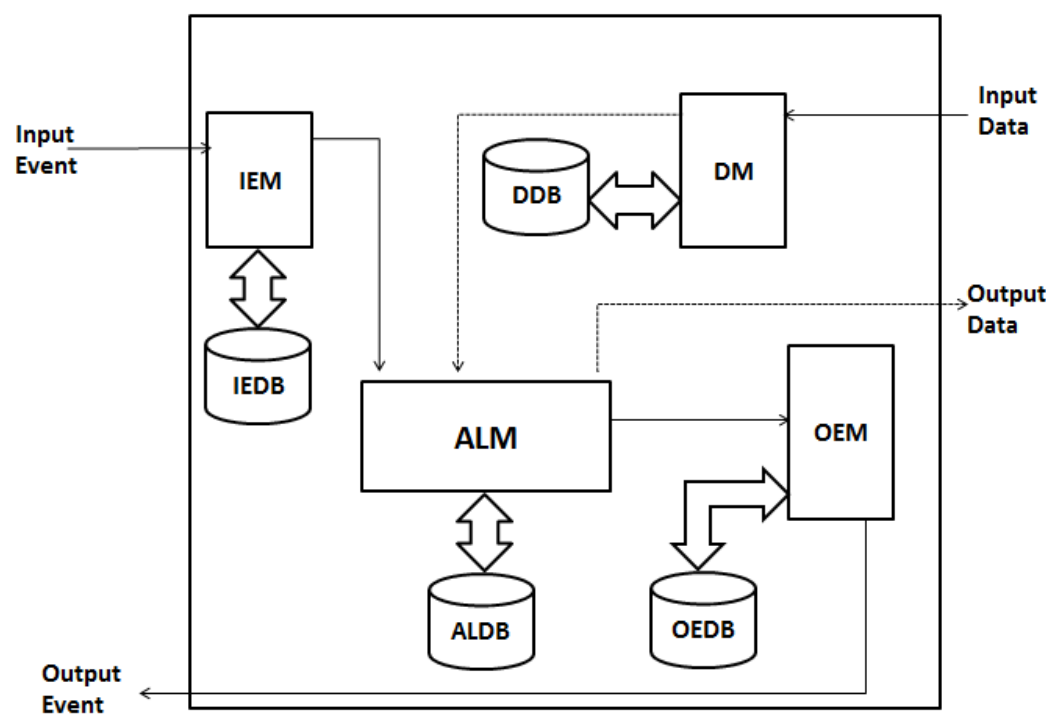

Fig. 2. RA2DL Architecture

Note that each reconfiguration scenario applied by $I E M$, $O E M, A L M$ and $D M$ defines the required sets of inputoutput events that activate corresponding algorithms of the component $C m p$ with well-defined values of data. A recon- 
figuration scenario defines a new execution model of $\mathrm{Cmp}$ to apply required services according to user requirements and also the evolution of the environment.

\section{Formalization}

We formalize the new RA2DL component by:

$$
C m p=(\beta, R)
$$

Where $\beta$ is Controlled Module of RA2DL to be described in the next section, and $\mathbf{R}$ is the Controller Module which is described in the three following levels:

1) First Level: Architectural Level (AL): Deals with the changes of the architecture of the RA2DL component when particular conditions are satisfied. In this case, it is possible to add, remove or also change the internal behavior of the component in IEM,OEM,ALM and $D M$. We denote by $\Psi_{C m p}$ the big set in $A L D B$ of all the possible algorithms involved in the different implementations of the component $C m p$, which is implemented at any particular time $t$ by a subset $\xi_{C m p}$ that represents the set of algorithms involved in a particular implementation $\xi_{C m p} \subseteq \Psi_{C m p}$. We model the architectural level $A L$ by a finite state machine $\mathbf{S}_{A L}$ such that each state of $\mathbf{S}_{A L}$ corresponds to a particular implementation of IEM,OEM, ALM and DM.

$\mathbf{S}_{A L}=\left(\Psi_{C m p}, \mathbf{O}, \delta\right)$, where:

- $\quad \mathbf{O}$ is a set of $n$ states in $\mathbf{S}_{A L}\left(\mathbf{O}=\left\{\mathbf{S}_{A L}^{i} \mid i \in 1\right.\right.$..n \} ),

- $\quad \delta$ is a state-transition function $\Psi_{C m p} \times \mathbf{O} \rightarrow \Psi_{C m p}$ $\mathrm{x} \mathbf{O}$.

The reconfigurataion in this level is supported by the Architectural Controller $A C$.

Running example. We distinguish three architectures of $R A 2 D L$ in the radar system as depicted in Fig.3

First architecture : when the weather is perfect, $(I E M=$ condition_weather $==$ $1)$, then we implement the RA2DL according to the first architecture (ASM1). Second architecture: when the weather is imperfect $(I E M=$ condition_weather $==0$ and $D M=$ wind_speed $<100 \mathrm{~km} / \mathrm{h})$, then we implement the RA2DL according to the second architecture ASM2. Third architecture: when the weather is perfect and the wind speed is high $(I E M=$ condition_weather $==0$ and $D M=$ wind_speed $>100 \mathrm{~km} / \mathrm{h}$ ), then we implement the RA2DL according to the third architecture ASM3.

2) Second Level: Composition Level (CL): This level keeps the same architecture in $C m p$ but just changes the composition of algorithms, input-output events in order to adapt the component to its environment. It is formalized by different Composition State Machines such that each one $C S M$ corresponds to a particular state in the Architecture Level $S_{A L}$. For each state $S_{A L}^{i}$ in $S_{A L}$, we define in the second hierarchical level (Composition Level CL) a particular state machine to be denoted by $S_{C L}^{i}$. Each state in

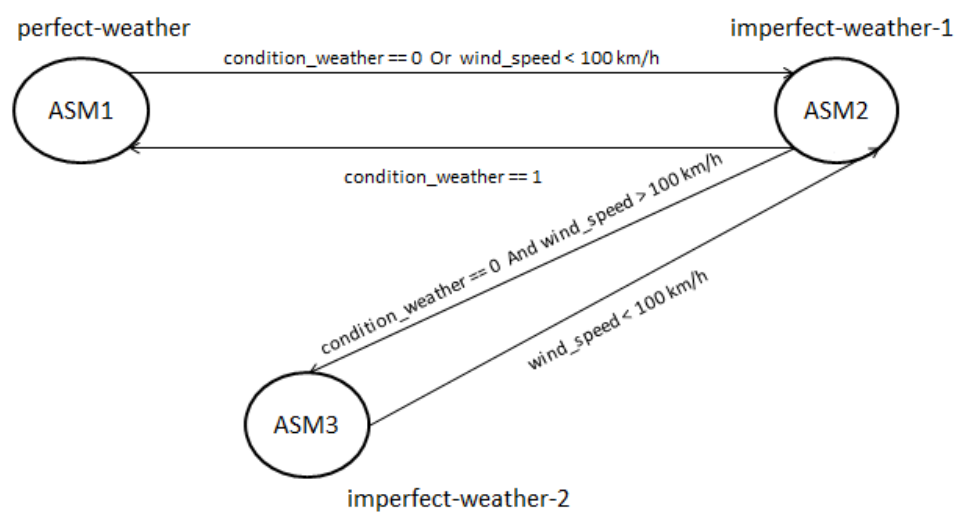

Fig. 3. First Architectural Level of RA2DL

$S_{C L}^{i, j}$ in $S_{C L}^{i}$ defines a particular composition of the subset of algorithms and input-output events. The reconfiguration in this level is supported by the Composition Controller $C C$.

Running example. We distinguish two compositions in the radar system for the first architecture (ASM1): the calculation of the angle can be done before or after the reception of signals (Fig.4). In this case, the component has two compositions CSM1 and CSM2 such that each one is characterized by the time intervals $T_{1}=20$ seconds and $T_{2}=60$ second.

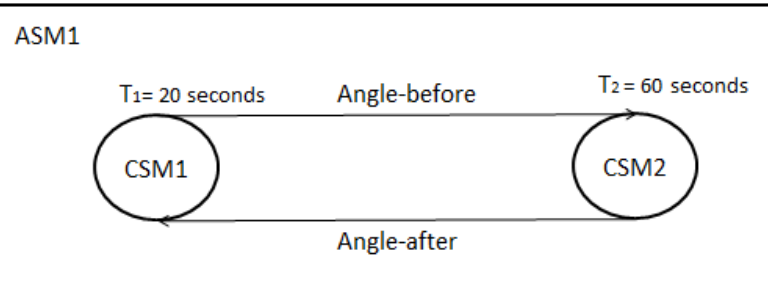

Fig. 4. Composition of ASM1

3) Third Level: Data level: This level deals with the light reconfiguration of data of the RA2DL component. It is formalized by a set of Data State Machines where each state of them corresponds to particular values of data. We define for each state $\mathbf{S}_{A L}^{i}$ of $\mathbf{S}_{A L}$ and for each state $\mathbf{S}_{C L}^{i, j}$ of $\mathbf{S}_{C L}^{i}$ a new state machine $\mathbf{S}_{D L}^{i, j, k}$ where each state corresponds to new values of data. The reconfiguration in this level is supported by the Data Controller $D C$.

Running example. In the radar system, if the weather problem occurs at run-time, we have to change the value of the parameter $C$ in $D M$ from $L_{-} R e s$ (DSM1) to $H_{-}$Res (DSM2). In this case, we will not be interested in any performance improvement but in the rescue of the whole system to guarantee a minimal level of safety.

Finally, this classification covers all possible reconfiguration forms to dynamically adapt the RA2DL component to 
the evolution in the environment according to user requirements.

\section{RA2DL behaviors}

To analyze the Controlled Module $(\beta)$ of a RA2DL, we characterize the corresponding algorithms by worst (resp, Best) case execution times $W C E T$ 's (resp, $B C E T$ ). Moreover, we consider that output events can be simultaneously sent or in exclusion according to user requirements. To validate the temporal behavior of a RA2DL component, we only focus on input events. We assume, in the rest of this paper, a complete synchronization between events and data. Indeed, when an event occurs in the corresponding input, all the associated data occur at the same time in the corresponding inputs. The different reconfiguration scenarios applied by the different controllers, define all possible behaviors in the $\beta$ Controlled Module. In this work, we specify these behaviors by a unique Behavior State Machine (denoted by $B S M$ ) where each state corresponds to a particular behavior of the RA2DL component.

Running example. We specify in Fig.5 the different behaviors of the controlled part that we can follow for all reconfigurations scenarios. We distinguish five branches of different behaviors. Branch 1 specifies the system behavior when Reconfiguration 1 is applied (e.g $s_{j}<C$ ), Branch 2 specifies the system behavior when Reconfiguration 2 is applied (e.g condition_weather $==1$ ), Branch 3 specifies the system behavior when Reconfiguration 3 is applied (e.g condition_weather $==0$ ). Branch 4 specifies the system behavior when Reconfiguration 4 is applied (e.g wind_speed > $100 \mathrm{~km} / \mathrm{h}$ ), and Branch5 specifies the system behavior when the 4Reconfiguration 5 or (e.g wind_speed $<100 \mathrm{~km} / \mathrm{h}$ ) is applied.

\section{Modelling AND VERIFICATION OF RA2DL}

We propose in this section the modelling and verification of RA2DL by using the UPPAAL tool [23]. We model the Controller Module of RA2DL by Nested State Machines such that the Architectural Level is specified by $A S M$ in which each state corresponds to a particular architecture of the component. Therefore, each transition of $A S M$ corresponds to an activation or desactivation of algorithms and input-output events. A state of $A S M$ corresponds to a particular state machine in the Composition Level denoted by $C S M$. This state machine specifies all the composition forms of algorithms and input-output events to be activated in this architecture state of the first level. A state of the Composition Level corresponds to a state machine in the Data Level $D S M$ that specifies all possible values of data in the RA2DL component. The Controller Unit applies automatically different run-time reconfiguration scenarios such that each one is denoted by Reconfiguration $i$ where $i \in[1.5]$.

Running example. We present in Fig.6 the nested state machines of RA2DL component in all levels of reconfiguration. The ASM state machine is composed of three states ASM1, ASM2 and ASM3 corresponding respectively to the first architecture (i.e. perfect weather), the second architecture (i.e. imperfect weadher) and the third architecture (i.e. perfect weather and wind speed is high). $A S M 1$ corresponds in the second level to the nested state machine CSM1 which is composed of two states CSM11 and CSM12 that specify respectively the cases of perfect and imperfect weather. ASM2 corresponds to the states CSM21, CSM22 that specify the wind speed, and CSM23 and CSM24 that specify the weather condition. ASM3 corresponds to the composition CSM31, CSM32 for the combination between weather and wind conditions. Finally $D S M$ specifies the reconfiguration of the data processing component.

In the RA2DL, all the forms of reconfigurations are given in Fig.7 which has five locations: Reconfiguration 1, Reconfiguration 2, Reconfiguration 3, Reconfiguration 4, and Reconfiguration 5 . We initially start by Reconfiguration 1 , which corresponds to a processing component when the condition $S_{-} j<C$ is assumed. In this case, the processing component reconfigures the parameter $C$ from $L \_R e s$ to $H_{-}$Res. If the weather situation is normal then the condition condition_weather $==0$ is satisfied. In this case, the radar system passes to the state Reconfiguration 3 after which the pulses are periodically sent from the antenna component by a thread component $E V \_T 2$ each $2 \mathrm{~ms}$ in this state. If wind_speed $>100 \mathrm{~km} / \mathrm{h}$ then the radar system passes to the state Reconfiguration 4 where the first motor component $M 1$ rotates by $45 \mathrm{tr} / \mathrm{mn}$ and the component Rotat 1 is executed to control the first motor. Otherwise when the condition wind_speed $<100 \mathrm{~km} / \mathrm{h}$ is satisfied then the radar passes to the state Reconfiguration 5 where the second motor component $M 2$ rotates in $30 \mathrm{tr} / \mathrm{mn}$ and the component Rotat2 is executed to control the second motor. The same thing, is repeated for the Reconfiguration 2 when the condition condition_weather $==1$ is satisfied.

In Fig.6, we present the automata of the controlled module describing the bevahior of the radar system represented by algorithms, input-output events/data. Fig.7 models all the reconfiguration to be performed by the controller module.

We check the correctness of the system's behavior after any reconfiguration scenario in order to avoid any unpredictable execution.

Running example. In the assumed radar component, we check simple reachability, safety, liveness and deadlock-free properties. The simple reachability properties are checked if a given location is reachable:

- $\boldsymbol{P 1}=A[] R A 2 D L .($ Reconfiguration2 or Reconfiguration3 or Reconfiguration4 or Reconfiguration5) :the radar system should work in all weather conditions,

- $\boldsymbol{P 2}=A[]$ RA2DL.Reconfiguration4.M1: The Motor M1 turns when the condition wind_speed $>100 \mathrm{~km} / \mathrm{h}$ is satisfied,

- $\boldsymbol{P 3}=A[] R A 2 D L$. Reconfiguration5.M2: The Motor $M 2$ turns when the condition wind_speed $<100 \mathrm{~km} / \mathrm{h}$ is satisfied. 


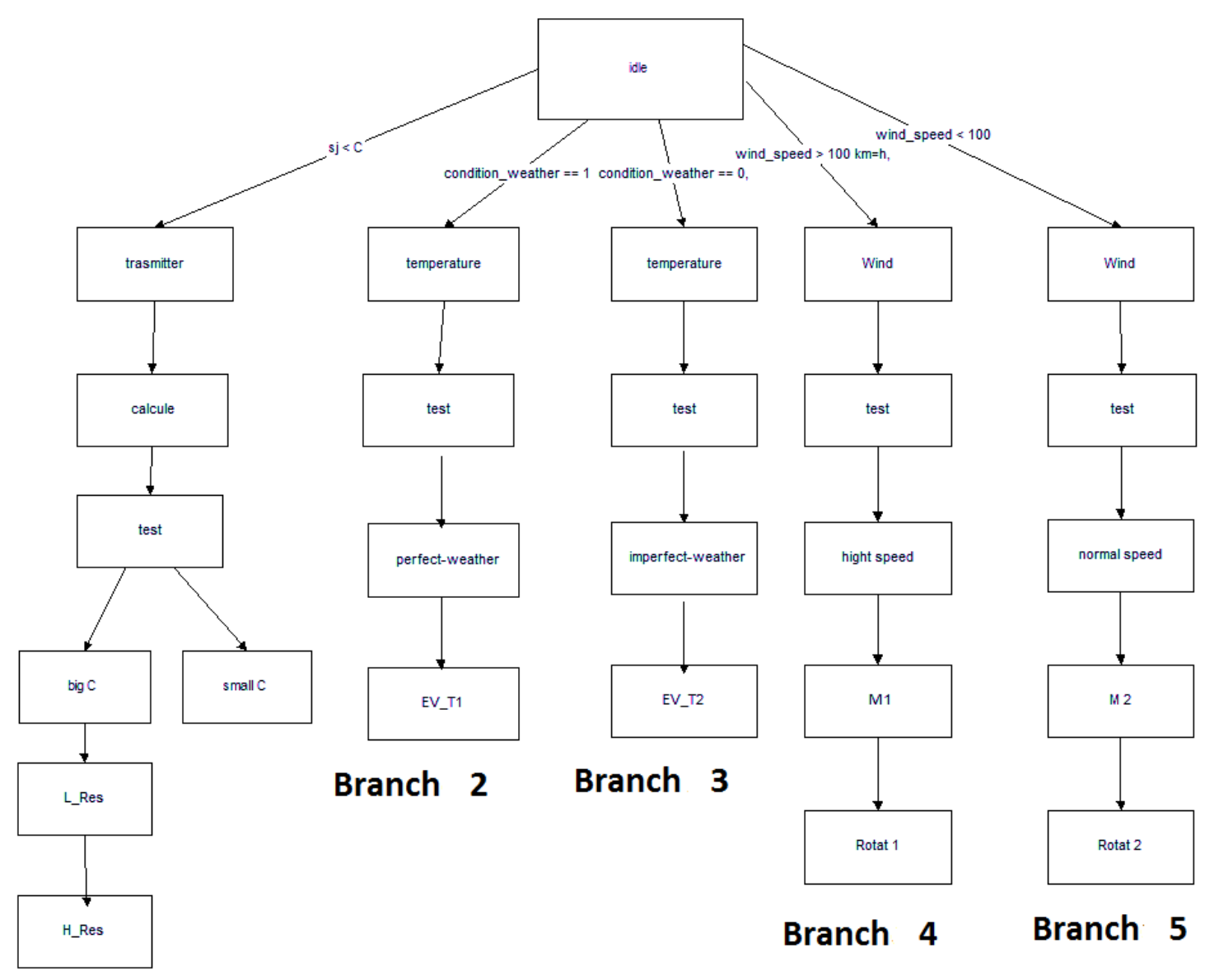

\section{Branch 1}

Fig. 5. Behaviors of the controlled module

The following safety properties must be held for all reachable states:

- $\boldsymbol{P 4}=A[] R A 2 D L$. Reconfiguration4. $r=45:$ In bad climate conditions the Motor M1 must rotate with a welldefined speed equal to $45 \mathrm{tr} / \mathrm{mn}$.

- $\boldsymbol{P 5}=A[] R A 2 D$ L.Reconfiguration5.r=30: In good climate conditions the Motor M2 must rotate with a welldefined speed equal to $30 \mathrm{tr} / \mathrm{mn}$.

The liveness properties are specified as follows:

- $\boldsymbol{P 6}=\quad A[](R A 2 D L . A S M 1$ implies $R A 2 D L$. Reconfiguration $3 . x<=2$ ) and (Radar.ASM2 implies RA2DL.Reconfiguration $2 . x<=6$ ): Bounded Liveness: A RA2DL will reconfigure the sending signal in maximum within 2 seconds in Reconfiguration 3 and 6 seconds in Reconfiguration2.

- $P 7=\quad R A 2 D L . R e c o n f i g u r a t i o n 3 \quad$ implies RA2DL.Reconfiguration4: Whenever wind_speed > $100 \mathrm{~km} / \mathrm{h}$, the corresponding M1 will eventually turn.

- $\boldsymbol{P 8}=\quad$ RA2DL.Reconfiguration3 implies RA2DL.Reconfiguration5: Whenever a wind_speed $<$ $100 \mathrm{~km} / \mathrm{h}$, the corresponding M2 will eventually turn.

\begin{tabular}{|c|c|c|c|}
\hline Property & Result & Time (sec) & Memory (Mo) \\
\hline P1 & Yes & 16.37 & 4.45 \\
\hline P2 & Yes & 4.48 & 4.03 \\
\hline P3 & Yes & 12.20 & 4.20 \\
\hline P4 & Yes & 10.34 & 4.20 \\
\hline P5 & Yes & 3.44 & $4 ; 03$ \\
\hline P6 & Yes & 8.50 & 4.20 \\
\hline P7 & Yes & 13.16 & 4.45 \\
\hline P8 & Yes & 7.12 & 4.20 \\
\hline P9 & Yes & 4.23 & 4.03 \\
\hline \multicolumn{3}{|c|}{ TABLE 1 } \\
\hline
\end{tabular}

EVALUATION OF THE VERIFICATION

The deadlock-free property is described as follows:

- $\boldsymbol{P 9}=A[] R A 2 D L$ not deadlock :the system is deadlockfree.

The verification of these properties is summarized in Table I. 

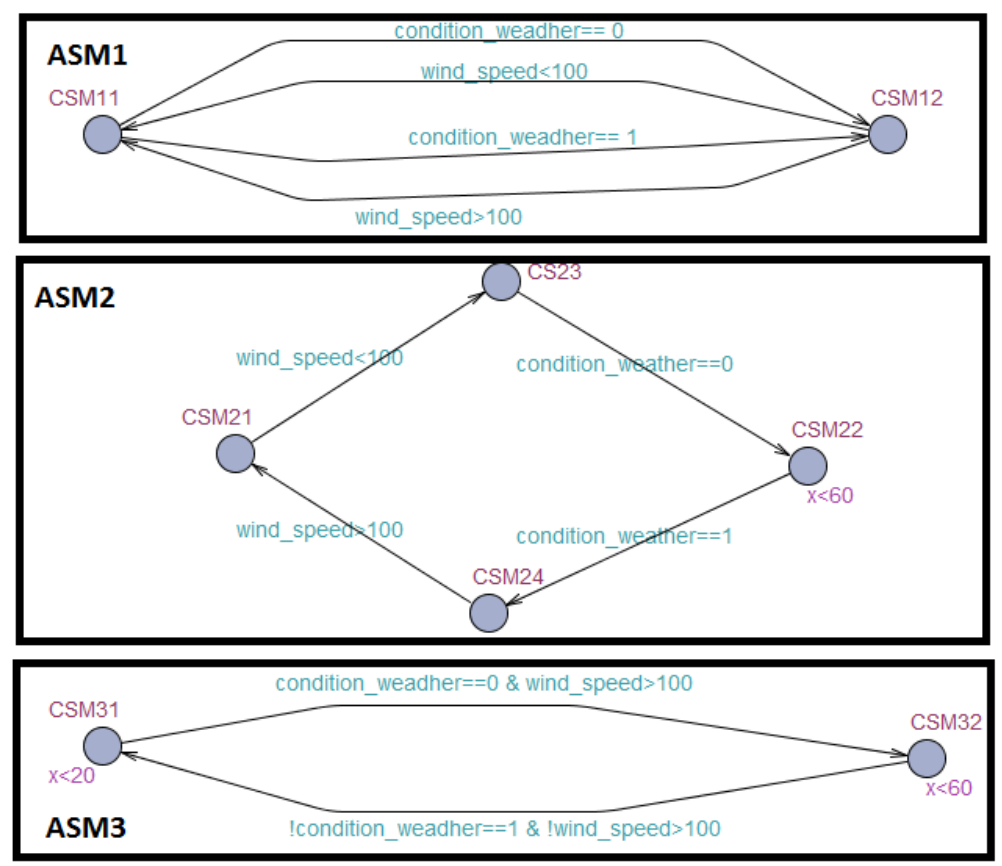

Fig. 6. Nested State Machines of RA2DL

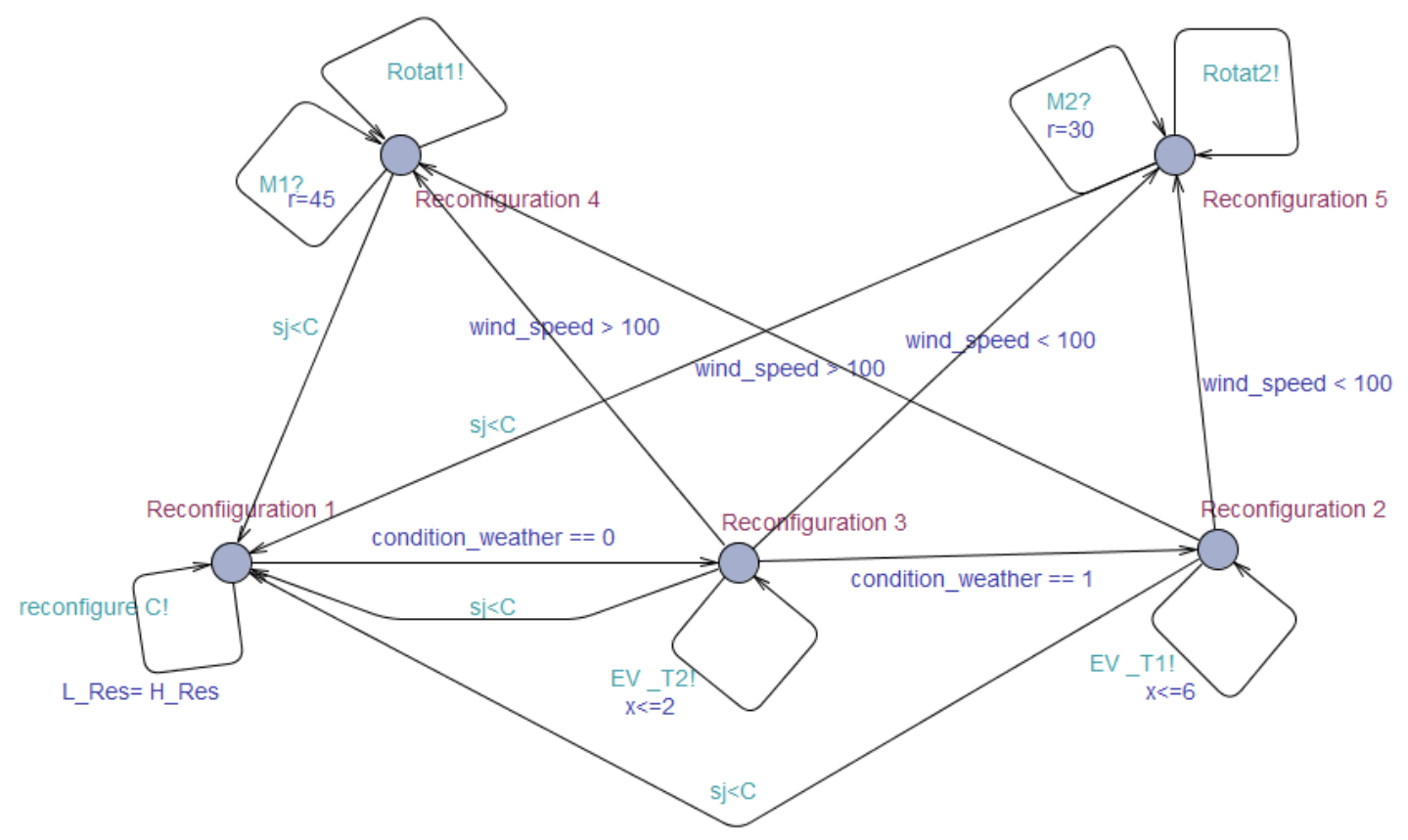

Fig. 7. Modeling of the Controller module 


\section{Simulation}

We present in this section the simulator RA2DLtool and the radar system that we developed in LISI Laboratory at INSAT Institute of University of Carthage in Tunisia. First, we present some interfaces of the simulator RA2DLtool. Second we show a simulation of the RA2DL-based radar system implemented in Arduino Uno microcontroller with ATMega32 processor ( 8 bits) and SRAM $2 \mathrm{~KB}$, the antenna is represented by an ultrasound sensor hc-SR04 and the motor is represented by Servomoteur df05bb with a power supply of $160 \mathrm{~mA}(4.8 \mathrm{~V})$, speed 0.17 seconds/60 degrees. The implementation and simulation of the radar system are represented in Fig.8.

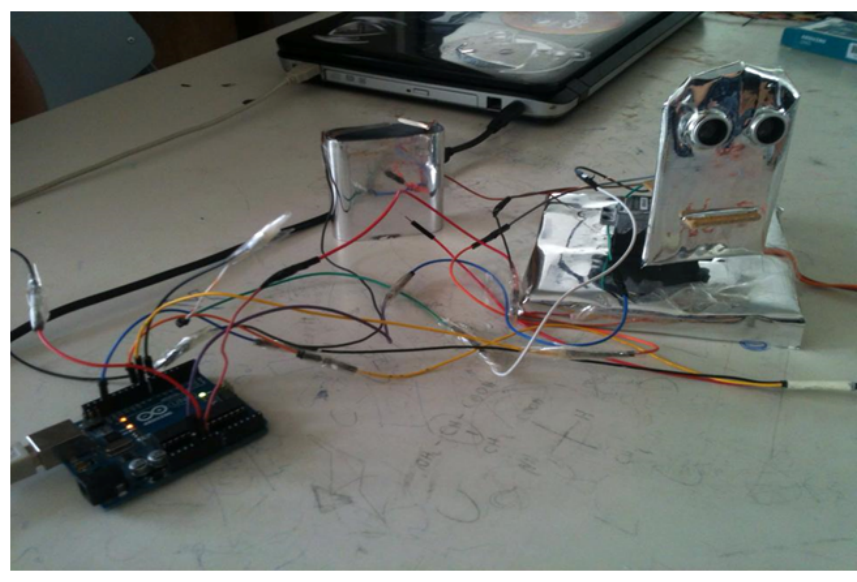

Fig. 8. Radar System

The $R A 2 D L$ tool offers the possibility to create all reconfiguration scenarios of the RA2DL component (addition,removal and update of algorithms, events and data) when any weather problem occurs (Fig.9).

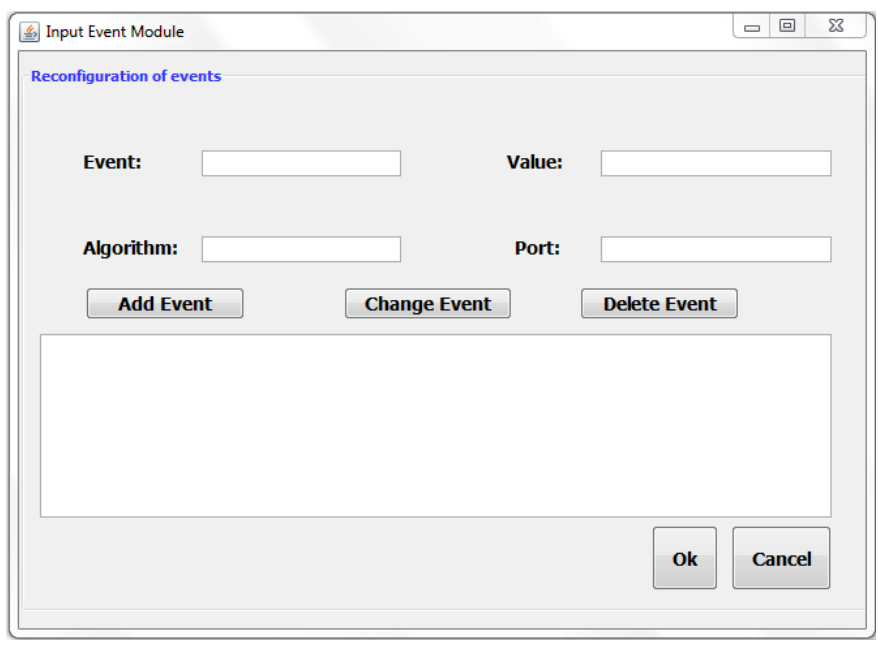

Fig. 9. Interface for Reconfiguration Architecture in IEM

Running example. In the radar system (Fig.11), we assume that the perfect-weather mode is applied. To verify the interaction between the controller and controlled modules when a problem imperfect-weather appears, we change the state of the rotor and antenna component. Consequently, the $A C$ decreases or changes the time of sending the signals, angles and rotations of the rotor. AC studies the feasibility of this new reconfiguration in order to accept the composition change of the system. In this case, the AC controller sends a final confirmation to officially apply this new reconfiguration. The result of this reconfiguration is displayed on the screen of radar as in Fig. 10.

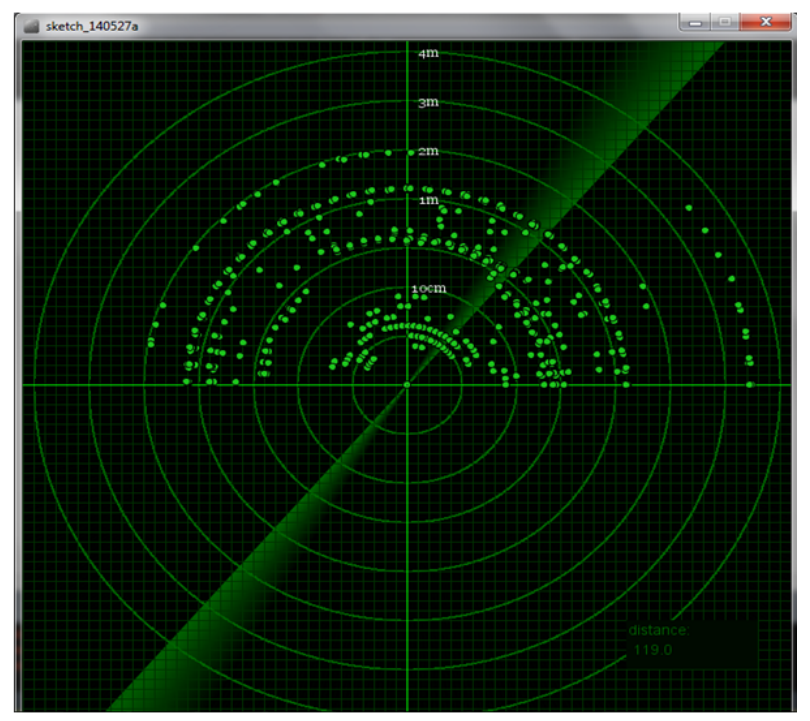

Fig. 10. Result after reconfiguration

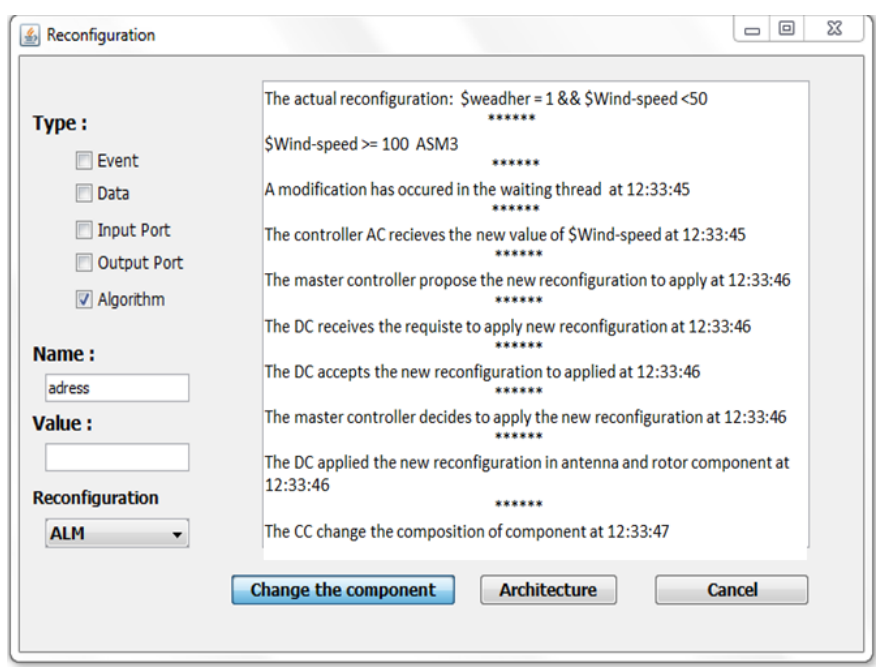

Fig. 11. Example of reconfiguration

The $R A 2 D L$ is a solution for the run-time reconfiguration of the AADL component in the radar system. By this solution the AADL component has become dynamic and flexible. None of the existing works has treated the dynamic reconfiguration of the AADL components as our method did.

\section{CONCLUSION}

The paper deals with new solutions for a required flexibility of adaptive control systems. It is applied to a radar system 
following the AADL language. We classify all possible reconfiguration scenarios of a component into three forms: The first deals with the component architecture, the second with the internal composition of algorithms as well as inputoutput events and the third with the reconfiguration of data. We propose a new concept named RA2DL to enrich the AADL Language by adding the flexibility criterion to its components. RA2DL is composed of a Controller module that allows all forms of reconfigurations, and a Controlled module that encodes all possible reconfigurable services to be offered by the component. The Controller module is modelled by Nested State Machines to control the complexity of the reconfiguration problem, whereas the Controlled module is modelled by a multi-branches state machines where each branch corresponds to a particular reconfiguration scenario. We plan in the future works to study the reconfiguration of several RA2DL components that should be coherent after any reconfiguration scenario to avoid any faults of interoperability. This work will be extended for the reconfiguration of distributed systems where new RA2DL components should be defined to allow feasible and coherent distributed reconfigurations on different devices.

\section{REFERENCES}

[1] C. Lozoya, M. Velasco, and P. Marti, "The one-shot task model for robust real-time embedded control systems," Industrial Informatics, IEEE Transactions on, vol. 4, no. 3, pp. 164-174, Aug 2008.

[2] Z. Peng, L. Ma, and F. Xia, "A low-cost embedded controller for complex control systems," in Embedded and Ubiquitous Computing, 2008. EUC '08. IEEE/IFIP International Conference on, vol. 1, Dec 2008, pp. 2329.

[3] C. Angelov, K. Sierszecki, and N. Marian, "Design models for reusable and reconfigurable state machines," in in L.T. Yang et al. (Eds.): Proc. of EUC 2005, LNCS 3824, 2005, pp. 152-163.

[4] M. N. Rooker, C. Sünder, T. Strasser, A. Zoitl, O. Hummer, and G. Ebenhofer, "Zero downtime reconfiguration of distributed automation systems: The \&\#949;cedac approach," in Proceedings of the 3rd International Conference on Industrial Applications of Holonic and Multi-Agent Systems: Holonic and MultiAgent Systems for Manufacturing, ser. HoloMAS '07. Berlin, Heidelberg: Springer-Verlag, 2007, pp. 326337.

[5] M. Khalgui, "Nces-based modelling and ctl-based verification of reconfigurable embedded control systems," Comput. Ind., vol. 61, no. 3, pp. 198-212, Apr. 2010.

[6] L. Zhu, X. Li, H. Ouyang, Y. Wang, W. Liu, and K. Shao, "Research on component-based approach load modeling based on energy management system and load control system," in Innovative Smart Grid Technologies - Asia (ISGT Asia), 2012 IEEE, May 2012, pp. 1-6.

[7] J. Lee and J.-S. Kim, "A methodology for developing component-based software with generation and assem- bly processes," in Advanced Communication Technology, 2004. The 6th International Conference on, vol. 2, Feb 2004, pp. 696-699.

[8] B. Baudry, F. Fleurey, J.-M. Jezequel, and Y. Le Traon, "Automatic test case optimization using a bacteriological adaptation model: application to net components," in Automated Software Engineering, 2002. Proceedings. ASE 2002. 17th IEEE International Conference on, 2002, pp. 253-256.

[9] F. Luders, "Adopting a software component model in real-time systems development," in Software Engineering Workshop, 2003. Proceedings. 28th Annual NASA Goddard, Dec 2003, pp. 114-119.

[10] Y. Liu, I. Gorton, A. Liu, and S. Chen, "Evaluating the scalability of enterprise javabeans technology," in Software Engineering Conference, 2002. Ninth AsiaPacific, Dec 2002, pp. 74-83.

[11] M. de Sousa, "Data-type checking of iec61131-3 st and il applications," in Emerging Technologies Factory Automation (ETFA), 2012 IEEE 17th Conference on, 2012, pp. 1-8.

[12] M. Khalgui, "Distributed reconfigurations of autonomous iec61499 systems," ACM Trans. Embed. Comput. Syst., vol. 12, no. 1, pp. 18:1-18:23, Jan. 2013.

[13] N. Medvidovic and R. N. Taylor, "A classification and comparison framework for software architecture description languages," IEEE Transactions on Software Engineering, vol. 26, no. 1, pp. 70-93, 2000.

[14] Y.-J. Seo, Y.-J. Song, and H.-Y. Jeong, “Acme-based connector interface considering component important factor," in SKG International Conference on Semantics, Knowledge and Grid (SKG 2005), 27-29 November 2005, Beijing, China. IEEE Computer Society, 2005, p. 54 .

[15] K. Palma, Y. Eterovic, and J. M. Murillo, "Extending the rapide adl to specify aspect oriented software architectures," in SEDE, 2006, pp. 170-167.

[16] R. Allen, R. Douence, and D. Garlan, "Specifying and analyzing dynamic software architectures," 1998.

[17] D. Kimpe, P. H. Carns, K. Harms, J. M. Wozniak, S. Lang, and R. B. Ross, "Aesop: Expressing concurrency in high-performance system software," in NAS, 2012, pp. 303-312.

[18] T. Vergnaud, L. Pautet, and F. Kordon, "Using the aadl to describe distributed applications from middleware to software components," in Proceedings of the 10th AdaEurope International Conference on Reliable Software Technologies, ser. Ada-Europe'05. Berlin, Heidelberg: Springer-Verlag, 2005, pp. 67-78.

[19] Y. Wang, D. Ma, Y. Zhao, L. Zou, and X. Zhao, "An aadl-based modeling method for arinc653-based avionics software," in Computer Software and Applications Conference (COMPSAC), 2011 IEEE 35th Annual, July 2011, pp. 224-229.

[20] T. teng Zhang, J. min $\mathrm{Wu}, \mathrm{L} . \mathrm{Qi}$, and H. yu Xu, "Architecture analysis and design language amp; har- 
mony system engineering process," in Digital Avionics Systems Conference (DASC), 2012 IEEE/AIAA 31st, Oct 2012, pp. 7D2-1-7D2-12.

[21] A. Prijic, Z. Prijic, D. Vuc-kovic, and A. Stanimirovic, "Aadl modeling of $\mathrm{m} 2 \mathrm{~m}$ terminal," in Microelectronics Proceedings (MIEL), 2010 27th International Conference on, May 2010, pp. 373-376.

[22] SAE, "Architecture analysis \& design language (standard sae as5506)," September 2004. [Online]. Available: http://www.sae.org

[23] J. Bengtsson, K. Larsen, F. Larsson, P. Pettersson, and W. Yi, "Uppaal;a tool suite for automatic verification of real-time systems." Secaucus, NJ, USA: SpringerVerlag New York, Inc., 1996, pp. 232-243.

[24] C. Yang, Y. Dong, F. Zhang, E. Ahmad, and B. Gu, "Formal semantics of aadl models with machinereadable csp," Computer and Information Science, ACIS International Conference on, vol. 0, pp. 565-571, 2012.

[25] P. Feiler, B. A. Lewis, and S. Vestal, "The sae architecture analysis \& design language (aadl) a standard for engineering performance critical systems," in Computer Aided Control System Design, 2006 IEEE International Conference on Control Applications, 2006 IEEE International Symposium on Intelligent Control, 2006 IEEE, 2006, pp. 1206-1211.

[26] G. Lasnier, L. Pautet, J. Hugues, and L. Wrage, “An implementation of the behavior annex in the aadl-toolset osate2," in Engineering of Complex Computer Systems (ICECCS), 2011 16th IEEE International Conference on, 2011, pp. 332-337.

[27] I. Perseil, L. Pautet, J. Rolland, M. Filali, D. Delanote, S. Baelen, W. Joosen, Y. Berbers, F. Mallet, D. Bertrand, S. Faucou, A. Zitouni, M. Boufaida, L. Seinturier, J. Champeau, T. Abdoul, P. Feiler, C. Mraidha, and S. Gerard, "An efficient modeling and execution framework for complex systems development," in Engineering of Complex Computer Systems (ICECCS), 2011 16th IEEE International Conference on, April 2011, pp. 317-331.

[28] M. Aniche, G. Oliva, and M. Gerosa, "What do the asserts in a unit test tell us about code quality? a study on open source and industrial projects," in Software Maintenance and Reengineering (CSMR), 2013 17th European Conference on, March 2013, pp. 111-120.

[29] V. Gaudel, A. Plantec, F. Singhoff, J. Hugues, P. Dissaux, and J. Legrand, "Enforcing software engineering tools interoperability: An example with aadl subsets," in Rapid System Prototyping (RSP), 2013 International Symposium on, Oct 2013, pp. 59-65.

[30] M. Kerboeuf, A. Plantec, F. Singhoff, A. Schach, and P. Dissaux, "Comparison of six ways to extend the scope of cheddar to aadl v2 with osate," in Engineering of Complex Computer Systems (ICECCS), 2010 15th IEEE International Conference on, March 2010, pp. 367-372.
[31] N. Pontisso and D. Chemouil, "Topcased combining formal methods with model-driven engineering," in Automated Software Engineering, 2006. ASE '06. 21st IEEE/ACM International Conference on, Sept 2006, pp. 359-360.

[32] J.-F. Tilman, "Building tool suite for aadl," in Architecture Description Languages, ser. IFIP The International Federation for Information Processing, P. Dissaux, M. Filali-Amine, P. Michel, and F. Vernadat, Eds. Springer US, 2005, vol. 176, pp. 197-207. [Online]. Available: http://dx.doi.org/10.1007/0-387-24590-1_13

[33] B. Zalila, L. Pautet, and J. Hugues, "Towards automatic middleware generation," in Object Oriented Real-Time Distributed Computing (ISORC), 2008 11th IEEE International Symposium on, May 2008, pp. 221-228.

[34] H. Liu and D. P. Gluch, "Formal verification of aadl behavior models: A feasibility investigation," in Proceedings of the 47th Annual Southeast Regional Conference, ser. ACM-SE 47. New York, NY, USA: ACM, 2009, pp. 36:1-36:6. [Online]. Available: http://doi.acm.org/10.1145/1566445.1566495

[35] A. Gharbi, M. Khalgui, and S. Ben Ahmed, "The embedded control system through real-time task," in Modeling, Simulation and Applied Optimization (ICMSAO), 2013 5th International Conference on, April 2013, pp. 1-8.

[36] P. Dissaux, "Using the aadl for mission critical software development," 2nd European Congress ERTS, EMBEDDED REAL TIME SOFTWARE, 2004.

[37] J. Hugues and F. Singhoff, "Développement de systèmes à l'aide d'aadl- ocarina/cheddar," in Tutoriel présenté à l'école d'été temps réel, Sep. 2009. 